\title{
Facilitated Discussion Template to Define Scenarios for a Grid Integration Study
}

Greening the Grid Grid Integration Studies Guide

Jessica Katz and Ilya Chernyakhovskiy 


\section{Background}

- The full guidebook on renewable energy grid integration studies can be found here.

- High-renewable energy (RE) scenarios represent the focus of a grid integration study and can be tailored to explore a range of potential futures or goals.

- This template provides examples of questions that can help the Technical Review Committee determine the high- and low-RE scenarios on which the grid integration study will focus.

- A scenario is one possible future electric generation system. A typical analysis has 3 to 5 key scenarios, which may vary by total RE generation penetration, locations of wind and solar generation, the ratio of solar to wind generation, transmission system expansion or other high-level assumptions about the future power system. 


\section{Objectives of a grid integration study}

1. Assess the impacts of adding a significant amount of variable renewable energy generation on the power system in a specific future year (i.e., 2030, 2050)

2. Evaluate strategies to improve wind and solar integration 
Objective 1: Assess the impacts of adding significant wind and solar on the power system

To meet objective 1 , it is important to define what is "significant" and what to compare this against.

- How much wind and solar? Where is it located?

- These are our high RE scenarios. In this example, we will choose four high RE scenarios to model.

- To assess the impacts of RE, we need to compare the high RE scenarios to a low RE scenario.

- The low RE scenario is our "base case."

Having multiple high RE cases enables the study to explore different combinations of RE. 


\section{How is the low RE base case different from today?}

The low RE base case will have new generation and transmission capacity to meet future load.

1. What new transmission should be included in the base case?

2. What new (conventional) generation capacity and retirements should be included in the base case?

3. In the base case, should the new conventional generation to meet future load be more flexible than today's, or the same mix as today?

- What will new generation look like? For example, will new natural gas plants use combined cycle or open cycle turbine technologies?

4. What changes to operating practices, if any, should be included in the base case (forecasting improvements, market changes, thermal turn-downs, etc.)?

5. Are there any other changes to power system hardware or operations that should be included in the base case?

6. What RE plants are $100 \%$ certain to be built, and should they be included in the base case (and all high-RE scenarios)? 
- What are the policy/system implications of a range of targets? E.g.,

- What is needed to achieve a $30 \%$ RE target?

- What is needed to achieve a $50 \%$ RE target?

-What is the best way to achieve a certain greenhouse gas (GHG) reduction?

-What are the policy/system implications for developing wind versus solar?

- Where should RE be developed?

- Closer to existing infrastructure? Best resource locations? 
Defining High RE scenarios

Step 2: What type of target is most meaningful?

Choose a target type:

1. RE (or variable RE) penetration level for a target future year

2. RE (or variable RE) capacity for a target future year

3. GHG emission reductions for a target future year 


\section{Defining High RE scenarios Step 3: How should the high RE scenarios vary?}

For example, develop 4 scenarios that are comparable.

1. Target level, e.g.,
a. $20 \%, 30 \%, 40 \%$ RE generation or capacity (how much variable RE?)
b. $5 \mathrm{GW}, 10 \mathrm{GW}$ wind/or and solar
c. $25 \%, 50 \%, 70 \%$ GHG reductions

2. Mix of wind and solar
a. High wind; moderate solar
b. High solar; moderate wind
c. Equal mix wind and solar

3. RE siting strategy
a. Best RE sites versus closest to load
- $\quad$ E.g., large-scale versus rooftop solar PV

b. Different distributions of regional targets
Do we want 1, 2, 3, or 4 targets?

- If we choose 4 targets, then the other categories (mix of wind/solar, $R E$ siting strategy) stay constant.

- If we choose 3 targets, then we can also choose one other variable from one category

- If we choose 2 targets, then we can also choose two other variables from one category

- If we choose 1 target, then we can also choose three other variables from one or two categories. 


\section{Base Case:}

No new renewables compared to 2014

\begin{tabular}{|l|l|c|c|}
\hline Base case & & Solar (GW) & Wind (GW) \\
\hline Scenario 1 & Medium RE & 20 & Current \\
\hline Scenario 2 & High Solar & 100 & 60 \\
\hline Scenario 3 & High Wind & 60 & 100 \\
\hline Scenario 4 & Very High RE & 150 & 100 \\
\hline
\end{tabular}

\section{2 target year across all scenarios}

Source: Palchak, David, et al. Greening the Grid: Pathways to Integrate 175 Gigawatts of Renewable Energy into India's Electric Grid, Vol. I--National Study. No. NREL/TP-6A20-68530. National Renewable Energy Lab.(NREL), Golden, CO (United States), 2017. https://www.nrel.gov/docs/fy17osti/68530.pdf 
Examples of scenario selection from other integration studies: Eastern U.S. Interconnection

\section{Base Case:}

No new renewables compared to 2012

\begin{tabular}{|l|c|c|}
\hline Medium RE & $\begin{array}{c}\text { Solar }(\% \text { of total } \\
\text { capacity) }\end{array}$ & $\begin{array}{c}\text { Wind (\% of total } \\
\text { capacity) }\end{array}$ \\
\hline Each region has to meet $30 \%$ target & $2 \%$ & $9 \%$ \\
\hline $\begin{array}{l}\text { Best resource used in whole } \\
\text { interconnection to meet 30\% target }\end{array}$ & $25 \%$ & $20 \%$ \\
\hline
\end{tabular}

\section{6 target across all scenarios}

$30 \%$ scenarios show comparison of two different potential paths of development. Medium RE shows the interim impacts that could be expected.

Source: Bloom, Aaron, et al. Eastern renewable generation integration study. No. NREL/TP-6A20-64472.

National Renewable Energy Lab.(NREL), Golden, CO (United States), 2016.

https://www.osti.gov/servlets/purl/1318192 
Why are comparison cases limited to a single future year (rather than multiple years)?

- The main modeling interest is RE penetration levels, how those build-outs vary, and what are their impacts on the power system

- The actual year that this occurs is less important. The scenarios may or may not happen in a specific year, and that is okay.

- If the study focuses on more years, then other assumptions become more important and could reduce confidence in the RE impacts

- Fuel prices, load growth, transmission development 


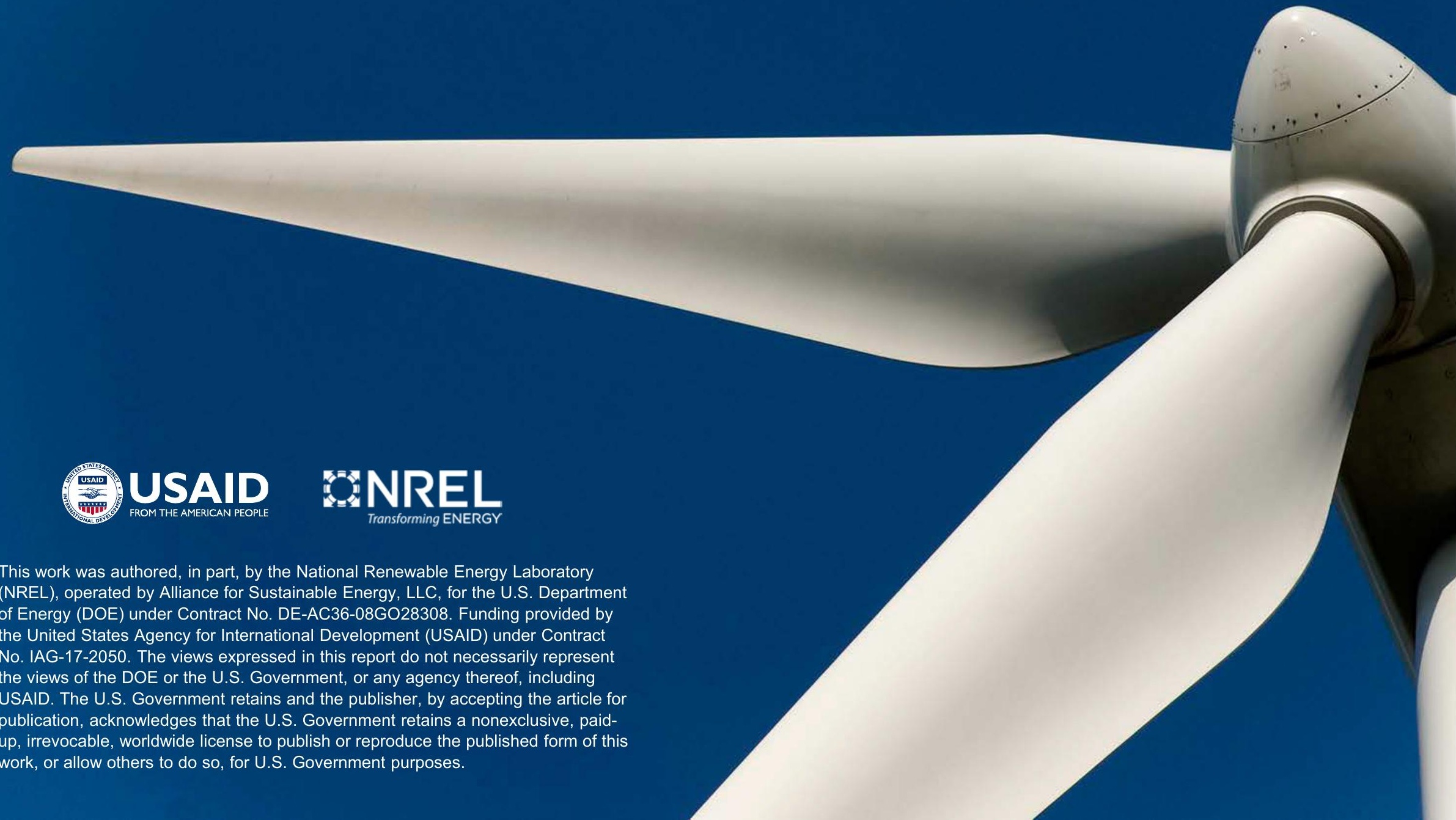

